\title{
Further Observations on a Pedigree of the Oral-Facial-Digital Syndrome
}

\author{
D. C. KERNOHAN and J. A DODGE \\ From the Department of Child and Preventive Dentistry; and the Nuffield Department of Child Health, \\ Queen's University, Belfast
}

We have previously reported a family with oral-facial-digital (OFD) syndrome in which 3 sisters were affected (Dodge and Kernohan, 1967). Their mother had only minor oral abnormalities and unusual fingerprints, but we suggested that she was also affected. Opportunity for further investigation of this family has occurred, and we here report our findings.

\section{Family Pedigree}

The family pedigree is given in Fig. 1.

The cases which we previously reported were IV.1, V.1, V.2, and V.3, as Cases 2, 3, 4, and 5, respectively. Since then we have been able to trace the mother of IV.1, and subsequently construct the remainder of the pedigree. A further daughter (V.4) has been born.

\section{Case Reports}

Case III.2. This patient was born in 1907. No evidence of any of the features of the syndrome was obtained in the antecedent or collateral family history.

On examination she was found to be edentulous and we were unable to find any record of her natural dentition.

Her tongue had a bifid tip and there was a large hamartoma on the right border (Fig. 2). She reported the removal of a similar lump from the left border in her childhood. On inspection a scar corresponding to that excision could be seen. Numerous small extra frenula were present in the vestibule between her lower lip and the alveolar ridge. There was a bony, hard swelling on the anterior palate. This had been present for at least 10 years, as it was recorded on the fitting surface of her denture.

She had mild ocular hypertelorism. There were no digital abnormalities, and no other physical features associated with this syndrome were found.

Fingerprints showed 6 ulnar loops (summed ridge count 55) and 4 arches (ridge count 0 ), but palm patterns were normal.

Received May 2, 1969.
She appeared to be of subnormal intelligence, and showed great emotional lability.

She had given birth to 3 children, 2 of whom were girls (IV.1 and IV.2); these 2 girls had different fathers.

Case IV.1. This patient was presented in our previous communication as Case 2. She was born in 1931 . On examination she had 2 supernumerary teeth which were similar in shape and location to permanent mandibular incisors. She had numerous small extra frenula in the lower vestibule.

She was also found to have arch patterns on all her fingerprints (ridge count 0 ). Her atd angles were $47^{\circ}$ and her palm patterns were normal. We had believed that she was an only child, but subsequently were able to trace her mother (III.2) and also to obtain the history of IV.2.

Case IV.2. This child was born in 1932 and died at the age of 8 months. We have not been able to establish any details of her cause of death, but she was a 'poor feeder' from birth. Her mother describes her as having had an abnormally shaped tongue, 'like a shamrock'. This appears to be a positive identification of the OFD syndrome.

Case V.1. Previously described as Case 3, she was born in 1955 . She presented with the following features of the syndrome: 'tongue-tie' (previously divided); bifid tongue tip; and multiple frenula in the lower labial sulcus, one of which traversed a gap in the alveolar ridge, becoming the lingual frenum. There was evidence of enamel hypoplasia but this was probably related to post-eruptive local oral conditions, and not genetically determined.

There was slight clinodactyly of both little fingers, but no other digital anomalies were seen.

Case V.2. Born in 1958, she was previously described as Case 4. She also was 'tongue-tied' at birth, and had surgical correction at an early age. Like her sister she had multiple frenal attachments in the lower labial sulcus and several in the upper canine region. The tongue had a bifid tip and there was a yellow fibrous rodule on the right edge. Both upper permanent 


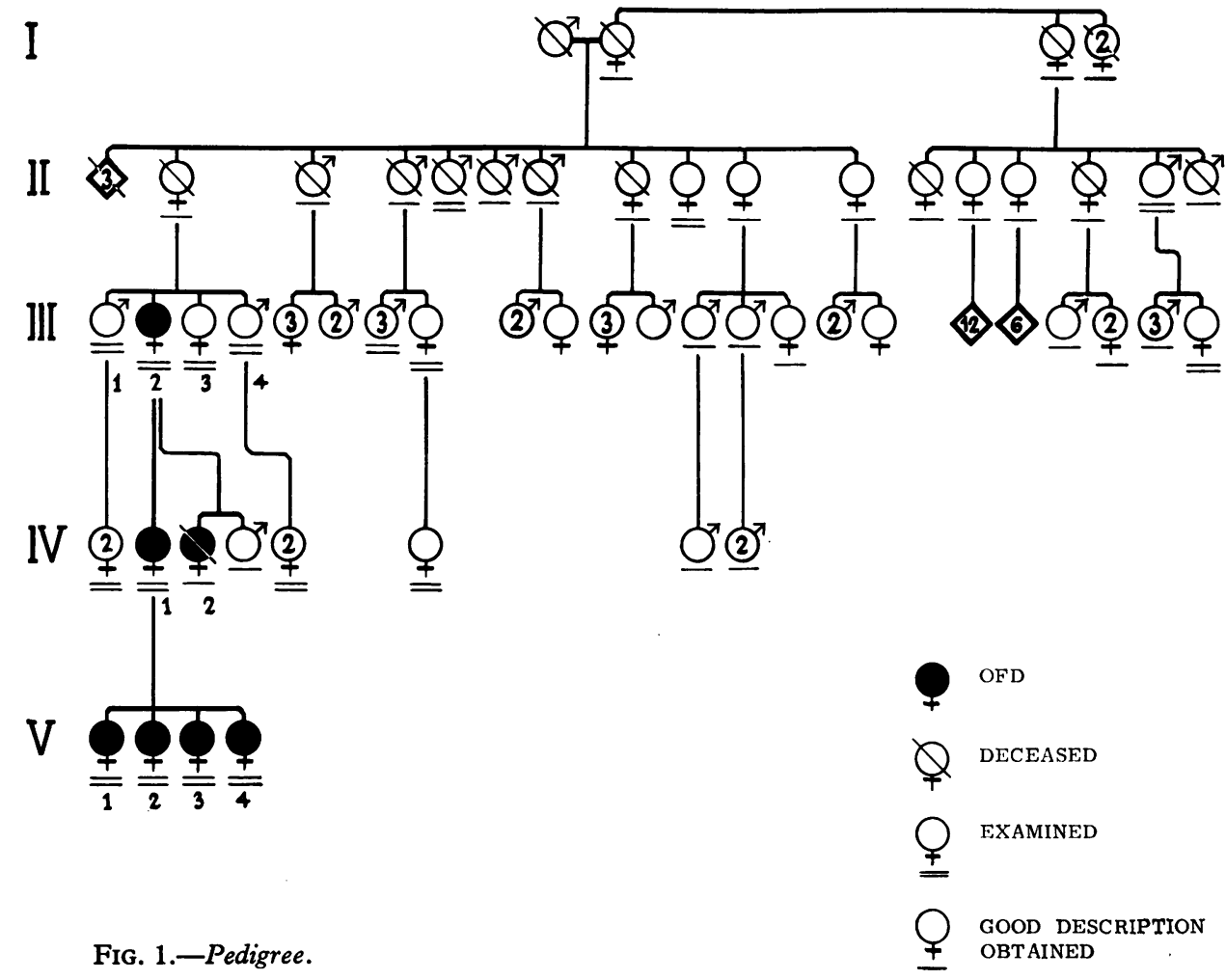

canines and both lower lateral incisors showed evidence of fusion with a supernumerary dental element. Facially she had slight epicanthus and frontal bossing. There was evidence of some alopecia, particularly in the frontal region.

Case V.3. Born in 1963. A nodule, about $1 \mathrm{~cm}$. across, was removed from her tongue at the age of 6 months. This proved to be composed of a core of fibro-fatty tissue in which there was some salivary

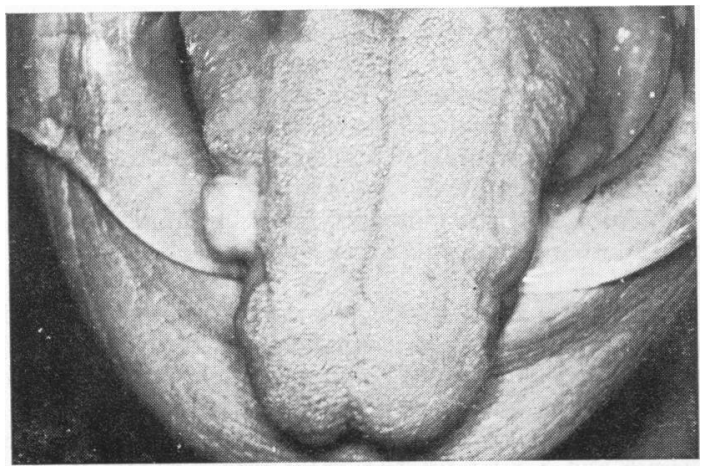

FIG. 2.-Tongue of Case III.2. gland tissue. The surface was stratified squamous epithelium.

She had marked frontal bossing, ocular hypertelorism, and marked epicanthic folds. Her nose was broad and flattened and her hair was sparse. Both hands showed short digits and clinodactyly. She had a lobulated tongue with hamartomata on the inferior surface between the lobules. Three of her lower incisors were absent. Her lingual frenum did not seem to impair the movements of her tongue

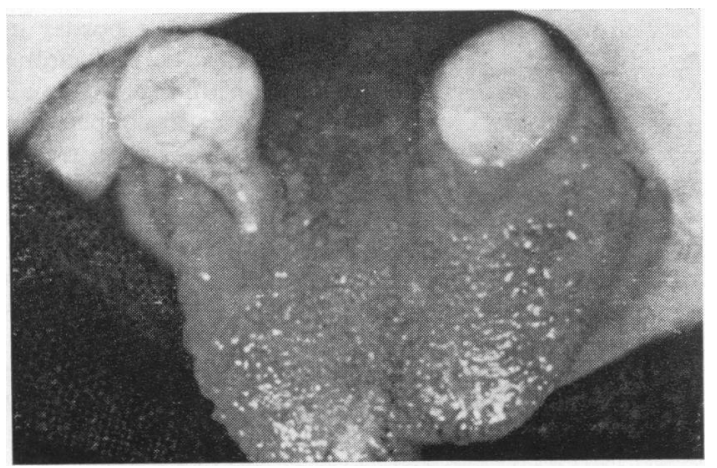

FIG. 3.-Tongue of Case V.4. 
although it was quite short and pronounced. There were numerous frenal attachments in the lower sulcus. She had a high-arched palate and there was evidence of a submucous cleft with lateral shelving of the mucoperiosteum.

Case V.4. This girl was born in July 1967. There was a faint midline scar of white fibrous tissue on the nose, and the left nostril was larger than the right. A similar faint vertical fibrous band was present to the right of the philtrum and another fibrous scar was seen in the middle of the upper lip, but there was no notching. The tip of the tongue was bifid, and 2 pedunculated hamartomata were present on its dorsal surface (Fig. 3). These were excised, and were histologically similar to those seen in other patients (Dodge and Kernohan, 1967). The lower alveolar ridge was divided by extra frenula. The palate was high-arched and there were bilateral longitudinal ridges. The fingers were normal and the toes showed no anomalies of shape though the fifth toes were as large as the fourth. No other malformations were noted.

\section{Discussion}

In our previous publication, we suggested that the minor abnormalities present in patient IV.1 were an expression of the OFD syndrome. There is no doubt, however, that without knowledge of the condition of her daughters, this diagnosis would not have been made as it was based only upon the presence of 6 lower incisors and 10 arch fingerprint patterns.

Though a large number of arches is an unusual dermatoglyphic finding, it has been described in a variety of disorders. These include E-trisomy (frequently), Klinefelter's syndrome, and partial C-trisomy. The latter occurrence is interesting in view of the association between partial C-trisomy and OFD syndrome in 2 patients of Patau et al. (1961). Arch patterns on all 10 digits have been found in a patient with $D / D$ translocation and in another with a short arm deletion of B4 (Hirschhorn and Shapiro, 1968); in pseudo-pseudohypoparathyroidism (Jongbloet and van Kempen, 1968); and in an apparently normal mother of a mongol infant (Crawfurd, 1968). The latter author concluded that 'preponderance of arches on digits may be due to chromosomal anomaly, single gene defect, or the operation, normal or abnormal, of multifactorial inheritance'. A universal digital arch pattern seems to be worthy of inclusion as a minor abnormality in our patient IV.2.

This patient's mother, III.2, who has now been examined, shows the OFD syndrome in unmistakable form. There is also good circumstantial evidence that the patient IV.2 who died in infancy showed characteristic features of the condition.
The fact that the 2 affected children of III.2 were born to different fathers is further strong evidence that OFD syndrome is transmitted as a dominant through the female. A similar occurrence has been noted by Doege and his associates (1964).

There is no evidence from verbal descriptions or photographs that any other female relatives were similarly affected, so it seems likely that a mutation arose in the case of III.2. However, the fact that the syndrome can evidently exist with minimal expression suggests that it could appear to miss several generations before reappearing. The patient of Gorlin, Anderson, and Scott (1961), described as a forme fruste, may have a different condition, i.e. misplaced tuberculum impar, but there can now be no doubt that patient IV.1 represents a true forme fruste. It appears that there are no certain diagnostic criteria by which such patients can be recognized. We therefore suggest that the diagnosis of OFD syndrome may be made on either of two grounds: (1) classical clinical features; or (2) minor oral or digital abnormalities with a positive family history of OFD syndrome.

\section{Summary}

Further data on a pedigree of the oral-facialdigital syndrome support the concept of extreme variability of expression of the syndrome. A true forme fruste has been identified. The syndrome may be diagnosed either when classical clinical features are present, or when minor oral or digital abnormalities coexist with a positive family history of the syndrome. 2 previously unreported features have been described, viz. pedunculated lingual hamartomata and abnormal fingerprint patterns.

\section{REFERENCES}

Crawfurd, M. d'A. (1968). Dermatoglyphics in partial C trisomies. Lancet, 1, 1195.

Dodge, J. A., and Kernohan, D. C. (1967). Oral-facial-digital syndrome. Arch. Dis. Childh., 42, 214.

Doege, T. C., Thuline, H. C., Priest, J. H., Norby, D. E., and Bryant, J. S. (1964). Studies of a family with the oral-facialdigital syndrome. New Engl. f. Med., 271, 1073.

Gorlin, R. J., Anderson, V. E., and Scott, C. R. (1961). Hypertrophie $d$ frenuli, oligophrenia, familial trembling and anomalies of the hand. ibid., 264, 486.

Hirschhorn, K., and Shapiro, L. R. (1968). Fingertip patterns. Lancet, 1, 1035.

Jongbloet, P. H., and van Kempen, C. (1968). Dermatoglyphics in partial $\mathrm{C}$ trisomies. ibid., 1, 1428.

Patau, K., Therman, E., Inhorn, S. L., Smith, D. W., and Ruess, A. L. (1961). Partial-trisomy syndromes. II. An insertion as cause of the O.F.D. syndrome in mother and daughter. Chromosoma (Berl.), 12, 573.

Correspondence to Dr. J. A. Dodge, Institute of Clinical Science, Grosvenor Road, Belfast 12, Northern Ireland. 